\title{
Optimieren Sie Ihre Krankenversicherung mit Sonderkonditionen für Ärzte
}

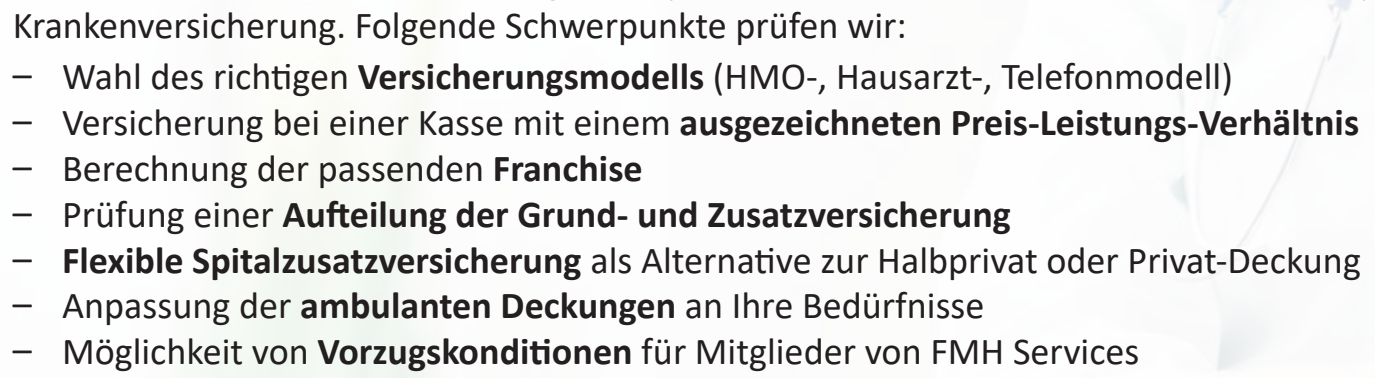
Krankenversicherung. Folgende Schwerpunkte prüfen wir:

- Wahl des richtigen Versicherungsmodells (HMO-, Hausarzt-, Telefonmodell)

- Versicherung bei einer Kasse mit einem ausgezeichneten Preis-Leistungs-Verhältnis

- Berechnung der passenden Franchise

- Prüfung einer Aufteilung der Grund- und Zusatzversicherung

- Flexible Spitalzusatzversicherung als Alternative zur Halbprivat oder Privat-Deckung

- Anpassung der ambulanten Deckungen an Ihre Bedürfnisse

- Möglichkeit von Vorzugskonditionen für Mitglieder von FMH Services

In unserem strukturierten Beratungskonzept suchen wir für Sie und Ihre Familie nach Optimierungspotential in Ihrer

\section{KRANKENVERSICHERUNG}

\section{TFMHSERVICES}

$\square$ Ich möchte einen Optimierungsvorschlag für meine Krankenversicherung (bitte Kopie der aktuellen Police beilegen)

Gewünschte Deckung

Gewünschter Versicherer

Folgende Deckungen sind mir wichtig: $\square \quad$ Allgemeine Spitalabteilung

$\square$ Private Spitalabteilung

$\square$ Bitte suchen Sie für mich das beste Angebot aus ODER

$\square$ Atupri

$\square$ Helsana-Gruppe

KPT

$\square$ Deckung im Ausland

$\square$ Freie Arztwahl

$\square$ Freie Spitalwahl

$\square$ Fitnesscenter-Abo

$\square$ Nichtpflichtmedikamente
Halbprivate Spitalabteilung

$\square$ Flexible Spitalversicherung

$\square$ CSS-Gruppe

innova

Komplementärmedizin

Transportkosten

Zahnspange

Brille / Kontaktlinsen

Vorsorgeuntersuchungen

Sind Sie mit einer Trennung der Grund- und Zusatzversicherungen einverstanden?

Kommt für Sie ein alternatives Versicherungsmodell in Frage?

Ja

$\square$ Nein

Bemerkungen

$\square$ Ich möchte eine persönliche Beratung. Bitte rufen Sie mich an.

Vorname / Name

Adresse

PLZ / Ort

Telefon Privat / Geschäft

Beste Zeit für einen Anruf

E-Mail-Adresse
INSURANCE

\section{Roth Gygax \& Partner AG}

Moosstrasse 2, 3073 Gümligen

Tel. 0319595000 - Fax 0319595010

mail@fmhinsurance.ch - www.fmhinsurance.ch

Die Roth Gygax \& Partner AG ist ein von der FMH Services Genossenschaft empfohlenes, rechtlich und wirtschaftlich selbstständiges Beratungsunternehmen. 